\title{
«SUPREMACISMO JUDICIAL» AL ESTILO ALEMÁN: LA SENTENCIA DEL TRIBUNAL CONSTITUCIONAL SOBRE EL PROGRAMA DE COMPRAS DE BONOS EN MERCADOS SECUNDARIOS'
}

\section{"Judicial supremacism" German style: the judgment of the constitutional tribunal on the public sector purchase programme}

SABRINA RAGONE

Universidad de Bolonia sabrina.ragone2@unibo.it

SUSANA RUIZ TARRÍAS

Universidad de Granada

starrias@ugr.es

Cómo citar/Citation

Ragone, S. y Ruiz Tarrías, S. (2020).

«Supremacismo judicial» al estilo alemán: la sentencia del Tribunal Constitucional sobre el programa de compras de bonos en mercados secundarios. Anuario Iberoamericano de Justicia Constitucional, 24(2), 421-448.

doi: https://doi.org/10.18042/cepc/aijc.24.13

\section{Resumen}

Este artículo ofrece un análisis crítico, contextual y comparativo de la sentencia del Tribunal Constitucional Federal alemán (TCFA) de 5 de mayo de 2020, en la que este último se opone frontalmente a la autoridad del Tribunal de Justicia de la UE (TJUE) y valora las actuaciones del Banco Central Europeo (BCE). Para comprender

1 El trabajo es el resultado de la reflexión conjunta de las autoras. Específicamente, los párrafos I y III fueron redactados por Sabrina Ragone; el II por Susana Ruiz Tarrías y el párrafo IV conjuntamente. 
la decisión, las autoras reconstruyen la evolución del papel del BCE en la gobernanza económica dentro de la eurozona, con particular referencia a las medidas de compra de bonos soberanos en los mercados secundarios estrenadas a raíz de la crisis financiera. Se centran en la jurisprudencia correspondiente del TJUE, cuestionada por el TCFA, y finalmente reflexionan sobre los conflictos entre ordenamientos nacionales y tribunales supra e internacionales a la luz del panorama comparado y global.

\section{Palabras clave}

Tribunal Constitucional Federal alemán; Tribunal de Justicia de la UE; Banco Central Europeo; compras de bonos en mercados secundarios; proporcionalidad.

\section{Abstract}

This article provides a critical, contextual and comparative analysis of the judgment rendered by the German Federal Constitutional Tribunal on the $5^{\text {th }}$ of May 2020, in which it challenged the authority of the Court of Justice of the EU (CJEU) and questioned the actions of the European Central Bank (ECB). To understand the decision, the authors examine the evolution of the role of the ECB within the economic governance of the Eurozone, with particular reference to the measures employed to purchase state bonds on secondary markets started as a result of the financial crisis. They focus on the corresponding jurisprudence of the CJEU, contested by the German Tribunal, and finally reflect on the conflicts between national law and supra - and international courts in light of the comparative and global panorama.

\section{Keywords}

German Federal Constitutional Tribunal; Court of Justice of the EU; European Central Bank; Purchase of State Bonds on Secondary Markets; Proportionality. 


\section{Sumario}

I. INTRODUCCIÓN. II. LA PARTICIPACIÓN DEL BANCO CENTRAL EUROPEO EN LA GOBERNANZA ECONÓMICA DE LA CRISIS ECONÓMICO-FINANCIERA DE LA EUROZONA: 1. Nuevos escenarios de participación y desarrollo de nuevas competencias. 2. La compra de bonos soberanos en los mercados secundarios en la jurisprudencia del tribunal de justicia. III. LA SENTENCIA DEL TRIBUNAL CONSTITUCIONAL FEDERAL ALEMÁN DE 5 DE MAYO DE 2020: DE LAS PALABRAS A LOS HECHOS: 1. Los argumentos del TCFA sobre falta de motivación y proporcionalidad. 2. ¿Un cortocircuito nacional? Ciudadanos y TCFA vs. instituciones democráticas. 3. Reacciones institucionales y críticas doctrinales. IV. IMPACTO SOBRE LA RESPUESTA A LA PANDEMIA POR COVID-19 Y RELEVANCIA PARA LOS CONFLICTOS ENTRE ESTADOS Y ORGANIZACIONES INTERNACIONALES MÁS ALLÁ DE EUROPA. BIBLIOGRAFíA.

\section{INTRODUCCIÓN}

La sentencia del Tribunal Constitucional Federal alemán (TCFA) de 5 de mayo de 2020 no representa un sorpresivo cambio de actitud del Alto Tribunal, ya que sus disonancias con respecto al derecho comunitario vienen de lejos, afectando tanto a su construcción conceptual como a sus contenidos. Así, este reciente pronunciamiento trae causa de la doctrina anterior relativa a la integración europea ( $\$$ III.1), al tiempo que constituye una consecuencia de la aplicación de la dogmática alemana.

Aun así, la misma ha sido más contundente de lo que cabía esperar conforme a la jurisprudencia más reciente, en la que el propio TCFA había abogado por una actuación europarechtsfreundlich, es decir, «amigable» hacia el derecho de la Unión Europea (UE), restringiendo su margen de intervención a los casos de violaciones de la competencia, por parte de la institución europea interesada, que tuvieran naturaleza manifiesta y estructural, y, más recientemente, 
reafirmando su deber de cooperación leal con el Tribunal de Justicia de la Unión Europea (TJUE) $)^{2,3}$.

Sin embargo, en este caso concreto, a pesar de reconocer la existencia de un debate doctrinal sobre los efectos económicos de las medidas enjuiciadas, y sobre los aspectos jurídicos más controvertidos (para. 113), el TCFA ha tomado una decisión perentoria en contra del TJUE y del Banco Central Europeo (BCE), que justamente se caracteriza por su inusitada "dureza» (Roca, 2020: 2847). El tono de la sentencia es muy poco «amigable», conteniendo una serie de acusaciones lapidarias que no buscan entrar en un diálogo con el TJUE, sino criticar tajantemente su actuación. Los jueces de Karlsruhe definen los razonamientos de este último como metodológicamente insuficientes (para. 153) y las motivaciones proporcionadas como objetivamente arbitrarias (para. 112) e insostenibles (para. 117), hasta vaciar de contenido y utilidad el principio de proporcionalidad (paras. 127 y 138).

Para llegar a comprender los precedentes, los contenidos y los efectos de esta sentencia, es indispensable partir del examen de cómo ha evolucionado, especialmente a raíz de la crisis económica de 2008, la participación del BCE en la gobernanza económica de la eurozona, hasta llegar a la consolidación de nuevas competencias, funciones innovadoras y medidas atípicas. En especial, la compra de bonos soberanos en los mercados secundarios se ha transformado en un mecanismo esencial de la política monetaria perseguida por el BCE y ha sido avalada por la jurisprudencia del TJUE en distintas ocasiones (\$ II). No obstante, la postura del TJUE no ha satisfecho los requerimientos y estándares del TCFA, que en la sentencia que aquí se comenta lamenta una carencia de motivación y proporcionalidad en las medidas de BCE y en el posterior análisis judicial realizado por el TJUE. Las reacciones de la doctrina y de los actores políticos han sido rápidas, numerosas y fuertemente críticas. Además, esta situación ha generado un cortocircuito nacional, en el que las decisiones tomadas por los actores políticos han sido cuestionadas por el TCFA y por ciudadanos particulares, más o menos conocidos, que han recurrido al mismo (\$ III).

La sentencia del TCFA abre nuevos escenarios en el debate europeo sobre las medidas adoptadas y a adoptar en respuesta a la pandemia por COVID-19, a la vez que se inserta en un contexto global de cuestionamientos a la autoridad de organizaciones internacionales, tanto en el viejo continente como en el nuevo mundo ( $\$ \mathrm{IV})$.

2 Véanse la decisión del Segundo Senado de 7 de junio de 2000 (2 BvL 1/97), disponible en: https://bit.ly/378TvUg; y la decisión del Segundo Senado del 6 de julio de 2010 (2 BvR 2661/06), disponible en: https://bit.ly/2HqVV7Z.

3 Sobre este concepto, Ziller (2010: 157). 


\section{LA PARTICIPACIÓN DEL BANCO CENTRAL EUROPEO EN LA GOBERNANZA ECONÓMICA DE LA CRISIS ECONÓMICO-FINANCIERA DE LA EUROZONA}

El riesgo para la estabilidad del euro a raíz de la crisis económicofinanciera de 2008, también tuvo como consecuencia un reforzamiento de la interacción no institucionalizada entre el BCE y los Estados miembros de la eurozona desconocida hasta entonces. De hecho, se afirma que la crisis económico-financiera de la eurozona «impulsó al Banco Central Europeo a otra dimensión» (Fromage et al., 2019: 4).

En efecto, el BCE ha desarrollado un papel relevante en la gestión de la crisis económico-financiera de la eurozona a través del cumplimiento de los objetivos que le encomienda el art. 282.2 del TFUE. El primero de ellos, calificado como "principal», consiste en mantener la estabilidad de precios; pero, inmediatamente a continuación se reconoce que, sin perjuicio del anterior, también prestará apoyo a las políticas económicas generales de la UE para contribuir a la consecución de los objetivos de esta.

Todo ello, desde la premisa del respeto por parte de las instituciones, órganos y organismos de la Unión y por los Gobiernos de los Estados miembros, a la independencia en el ejercicio de las funciones que el BCE tiene asignadas según el art. 282.3 TFUE.

Sin embargo, su presencia en nuevos foros, la asunción de nuevas prerrogativas y la utilización de instrumentos heterodoxos en el terreno de la estricta política monetaria, con claras repercusiones e interacciones con la política económica resulta criticada en la era postcrisis, no solo desde el punto de vista de la independencia del $\mathrm{BCE}^{4}$, sino también desde la perspectiva de su responsabilidad (Fromage et al., 2019, entre otros) ${ }^{5}$, o de su déficit democrático (Hinarejos, 2015; Högenauer y Howarth, 2019).

4 La independencia del BCE no ha sido nunca un tema pacífico en la doctrina, véanse, entre otros, Amtenbrink (2019), Hahn (1991), De Haan et al. (2004), Duisenberg (2005), Hinarejos (2015), Smits (1991 y 2008).

5 Una responsabilidad que en el Report on Economic and Monetary Union in the European Community (conocido como Informe Delors), en 1989, aparecía como uno de los dos rasgos del BCE junto a su independencia En dicho informe la responsabilidad se concretaba en la presentación por el BCE de un Informe anual al Parlamento Europeo y al Consejo, además de que su presidente pudiera ser invitado por estas instituciones para ser informadas. Se requería, también, una supervisión de la administración del sistema monetario llevada a cabo con independencia de los órganos comunitarios, por ejemplo, por un Consejo de supervisión (Committee for the Study of Economic and Monetary Union, 1989: 22). 
Un cuestionamiento que tiene como trasfondo la propia estructura que ha venido sustentando a la Unión Económica y Monetaria Europea desde el Tratado de Maastricht, pues la integración económica sin una integración política solo es posible si la política y la economía se mantienen lo más alejadas posible, de modo que la despolitización del proceso de decisión en la UE es «el precio que tenemos que pagar» para preservar intacta la soberanía nacional (Majone, 1998: 7) ${ }^{6}$.

Sin embargo, las previsiones sobre política monetaria de los Tratados UE (arts. 127.1 y 282.2 TFUE; 13 TUE), y el art. 2 de los Estatutos del Sistema Europeo de Bancos Centrales, se refieren a los «objetivos» y no tanto a los «instrumentos» de dicha política (Jędrzejowska-Schiffauer y Schiffauer, 2016: 200).

De hecho, los tratados no definen la expresión "política monetaria», y en virtud del tenor del art. 127.1 TFUE puede entenderse que el BCE está legitimado para apoyar las políticas económicas generales dentro de la Unión Europea y no solo de la Unión Europea, en la medida en que no se comprometa el objetivo de la estabilidad de los precios (Simon, 2015: 1029).

De ahí que la intervención del BCE en relación con los Estados miembros de la eurozona también pueda ser interpretada en el sentido de propiciar una

No obstante, los términos del actual art. 284.3 TFUE resultan significativamente más amplios, en tanto que el Informe anual se presenta sobre las actividades del SEBC y sobre la política monetaria del año precedente y del año en curso al Parlamento Europeo, al Consejo, a la Comisión y al Consejo Europeo, y el Consejo y el Parlamento Europeo podrán llevar a cabo un debate sobre el mismo. Además, el presidente y los miembros del Comité Ejecutivo del BCE, por iniciativa propia o a requerimiento del Parlamento Europeo, podrán ser oídos por las comisiones competentes. Unas previsiones que se completan en el art. 15 del Protocolo núm. 4 Sobre los Estatutos del Sistema Europeo de Bancos Centrales y del Banco Central Europeo.

6 Por su parte, Moravcsik (2002) entiende que el carácter tecnocrático de las políticas de la Unión constituye uno de los beneficios de la despolitización. En respuesta a ambos, Follesdal y Hix (2006).

En relación con esta polémica, cabe recordar que el Informe Delors (Committee for the Study of Economic and Monetary Union, 1989: 23), contemplaba previsiones en el ámbito económico que, a día de hoy, siguen sin ser implementadas en el derecho originario. En concreto, estimaba: «The new Treaty would therefore not have to determine the mandate, estatus and structure of a new institution but have to provide for additional or changed roles for the existing bodies in the light of the policy functions they would have to fulfil in an economic and monetary union. It would have to specifically define these changes and determine the areas in which decision-making authority would have to be transferred from the national to the Community level».

7 Véase la sentencia TJUE de 27 de noviembre de 2012, asunto C-370/12, Pringle, para. 53. 
«interacción» o un «diálogo» entre los bancos centrales y las instituciones políticas, con el objetivo de promover la coordinación de las decisiones sobre la política económica de los Estados miembros con la política monetaria del BCE (Beukers, 2013: 1581-1582).

\section{NUEVOS ESCENARIOS DE PARTICIPACIÓN Y DESARROLLO DE NUEVAS COMPETENCIAS}

En efecto, al margen de las previsiones expresas contempladas en los tratados ${ }^{9}$, la crisis de la eurozona ha reforzado las relaciones del BCE con los actores políticos, por ejemplo, a través de su participación en distintos foros de discusión sobre aspectos de política económica.

Como el Diálogo Macroeconómico creado en 1999, donde el BCE, junto al Consejo, la Comisión y los interlocutores sociales, llevan a cabo una reunión informal dos veces al año con el fin de fortalecer la comprensión mutua entre los encargados de adoptar decisiones en diferentes esferas de la política económica (ECB, 2010: 81).

Del mismo modo, el BCE interviene ahora en la Cumbre del Euro, reunión institucionalizada por los jefes de Estado y de Gobierno de la eurozona en 2011, y elevada a rango internacional en el año siguiente por el art. 12.1 del TECG (al tiempo que participa en las minicumbres, totalmente informales, previas a su celebración). Además, el presidente del BCE puede participar como observador en el Consejo de Gobernadores del MEDE (art. 5.3 del Tratado MEDE), y es miembro, desde 2010, junto a los representantes de los Estados miembros y el presidente del Consejo, del Grupo de Trabajo (Task Force) sobre la Gobernanza Económica (Conclusiones CE, 2010: 6).

8 Analizando los efectos de la adopción de la Unión Económica y Monetaria en los procesos de cooperación entre órganos con competencias en materia de política monetaria y presupuestaria, Bini Smaghi y Casini (2000) sugerían que en el tránsito a la moneda única, se había quedado en el camino la mejora del diálogo y la cooperación entre las autoridades monetarias y presupuestarias.

9 El art. 284.3 TFUE prevé un diálogo entre el BCE y el Parlamento Europeo a partir de la presentación por el primero del Informe Anual; el art. 284.1 TFUE contempla la posibilidad de que el Presidente del Consejo y un miembro de la Comisión, participen en las reuniones del Consejo de Gobierno del BCE sin derecho de voto pero con la capacidad para presentar iniciativas para su deliberación; el art. 284.2 TFUE regula la presencia del Presidente del BCE en las reuniones del Consejo cuyo objeto tenga relación con el SEBC; y el Protocolo núm. 14 del Tratado de Lisboa dispone que en las reuniones del Eurogrupo para examinar cuestiones relacionadas con la moneda única, se invitará al BCE a participar. 
Otra de las vías de interacción entre el BCE y las instituciones políticas, proviene de la utilización de sus competencias consultivas a través de opiniones y dictámenes en distintos procedimientos, como los previstos en los arts. 127.4 y 282.5 TFUE, sobre propuestas de la Unión que entren en su ámbito de competencias. Una función que, como consecuencia de la crisis económico-financiera, ejerce también en el ámbito del marco normativo de la UE para reforzar la gobernanza de la eurozona, entre otros instrumentos, a través del Two-Pack y el Six Pack ${ }^{10}$.

Asimismo, el BCE forma parte de la conocida como troika (BCE, Comisión Europea, FMI), en cuyo contexto desarrolla nuevas funciones relacionadas con la política económica de los Estados de la eurozona.

Concretamente, en el ámbito del Tratado MEDE, la Comisión, en coordinación con el BCE y conjuntamente con el FMI, negocia con el Estado miembro el Memorándum de Entendimiento en el que se define la condicionalidad asociada a la asistencia financiera (art. 13.3), y también en coordinación con el BCE conjuntamente con el FMI, vela por su cumplimiento (art. 13.7) ${ }^{11}$.

Junto a estas intervenciones en el ámbito de la política económica de la eurozona, el $\mathrm{BCE}$ ha asumido una función de vigilancia en la supervisión reforzada y en las misiones de control en el marco jurídico de la gobernanza económica de la UE que, combinada con su participación en la Troika, le confiere una importante capacidad de influencia sobre las reformas de las políticas económicas de los Estados miembros.

En este contexto, el BCE podrá ser invitado por la Comisión a participar en las misiones de supervisión llevadas a cabo en un Estado miembro de la eurozona o del MTCII (Mecanismo de Tipos de Cambio II) ${ }^{12}$; también

10 El Dictamen del BCE de 7 de marzo de 2012 sobre el reforzamiento de la gobernanza económica de la eurozona, en el marco del Two Pack (CON/2012/18), reconoce expresamente su fundamento en los arts. 127(4) y 282(5) del TFUE (DOUE C 141/7, de 17.5.2012).

11 La intervención de la troika para la negociación del Memorándum de Entendimiento que fijaba las condiciones de la asistencia financiera al Estado miembro, estaba prevista en el art. 2.1(a) del Acuerdo Marco de la Facilidad Europea de Estabilización Financiera (FEEF).

12 Art. 11.2 y 3 del Reglamento (UE) 1175/2011, del Parlamento Europeo y del Consejo, de 16 de noviembre de 2011, sobre el refuerzo de la supervisión de las situaciones presupuestarias y a la supervisión y coordinación de las políticas económicas.

El Mecanismo de Tipos de Cambio fue establecido mediante Resolución del Consejo Europeo de Ámsterdam (97C 236/03), de 16 de junio de 1997 (DOUE C 236/5, de 2.8.1997), con la finalidad de crear un sistema entre los Estados pertenecientes a la eurozona y también Estados no integrados en la misma, que permitiera garantizar la 
intervendrá, en coordinación con la Comisión, en las misiones de supervisión reforzada de los Estados de la eurozona o Estados miembros del MTC II a los que se abra un procedimiento de déficit excesivo, y en misiones de evaluación de riesgos para la situación económica en un Estado miembro de la eurozona o del MTC II $^{13}$. Del mismo modo, el BCE podrá ser invitado, en su caso, por la Comisión, a formar parte de las misiones de supervisión reforzada en Estados que hayan sido destinatarios de recomendaciones por desviaciones significativas en la trayectoria de ajuste presupuestario ${ }^{14}$.

Por su parte, la Comisión, en coordinación con el BCE y, en su caso, con el FMI, llevará a cabo misiones periódicas de evaluación en el Estado miembro sometido a supervisión reforzada; en los mismos términos, el BCE participará en el seguimiento de los progresos de un Estado miembro en la aplicación del programa de ajuste macroeconómico, y en las misiones periódicas de evaluación posteriores a la aplicación del programa ${ }^{15}$.

Junto a estas actuaciones, el BCE también ha implementado otras operaciones no convencionales, como las medidas de apoyo al crédito con el fin de proporcionar liquidez a los Bancos Centrales nacionales en casos de emergencia (provisión de liquidez de urgencia, emergency lending assistance, también conocidas como operaciones de acarreo, carry-trade) ${ }^{16}$, proporcionando las condiciones para que el Estado miembro en cuestión adopte las reformas exigidas por la condicionalidad gracias a la compra de bonos

estabilidad de los tipos de cambio entre las diferentes monedas al inicio de la Unión Económica y Monetaria en 1999. Posteriormente, el BCE firmó un Acuerdo (2006/C 73/08), de 16 de marzo de 2006, con los bancos centrales nacionales de los Estados miembros que no forman parte de la zona del euro por el que se establecen los procedimientos de funcionamiento del mecanismo de tipos de cambio de la tercera fase de la unión económica y monetaria (DOUE C73/21, de 25.3.2006), que pasan, en consecuencia, a integrarse como participantes el MTC II.

13 Arts. 9.3 y 13.2-3, del Reglamento (UE) 1176/2011, del Parlamento Europeo y del Consejo, de 16 de noviembre de 2011, relativo a la prevención y corrección de los desequilibrios macroeconómicos.

14 Art 11.3 del Reglamento (UE) n. 1177/2011, del Consejo, de 8 de noviembre de 2011, relativo a la aceleración y clarificación del procedimiento de déficit excesivo.

15 Arts. 3.5, 7.4 y 14.3 del Reglamento (UE) 472/2013, del Parlamento Europeo y del Consejo, de 21 de mayo de 2013, sobre el reforzamiento de la supervisión económica y presupuestaria de los Estados miembros de la zona euro cuya estabilidad financiera experimenta o corre el riesgo de experimentar graves dificultades.

16 Estas funciones estarían comprendidas en las competencias residuales que mantienen los bancos centrales nacionales según las previsiones del art. 14.4 del Protocolo (núm. 4) sobre los Estatutos del Sistema Europeo de Bancos Centrales y del Banco Central Europeo, anejo al Tratado de Lisboa. 
soberanos por los propios bancos nacionales que, previamente, han obtenido liquidez a bajo precio del $\mathrm{BCE}^{17}$.

El conjunto de actuaciones descritas pone de manifiesto la "poderosa posición» adquirida por el BCE mediante la combinación del ejercicio de sus funciones, con su papel como "conegociador» y «observador» junto a la Comisión Europea (Beukers, 2013: 1594).

\section{LA COMPRA DE BONOS SOBERANOS EN LOS MERCADOS SECUNDARIOS EN LA JURISPRUDENCIA DEL TRIBUNAL DE JUSTICIA}

En todo caso, las intervenciones más polémicas de todas las desplegadas por el BCE a raíz de la crisis económico-financiera de 2008 son, con mucho, los programas de compra de bonos soberanos en los mercados secundarios (o indirectos).

El fundamento de dicha actuación cabe encontrarlo en el primer guion del art. 127.2 TFUE y en el art. 18.1 de los Estatutos del SEBC y del BCE, que le habilitan para operar en los mercados financieros comprando y vendiendo directamente, entre otros activos, instrumentos financieros (como es el caso de los bonos) $)^{18}$.

Al ser negociados en mercados secundarios, la compra de estos bonos constituye una operación financiera que no contraviene, en principio, la prohibición de que el $\mathrm{BCE}$ o los bancos centrales nacionales adquieran directamente instrumentos de deuda de los Gobiernos centrales, de autoridades regionales o locales u otras autoridades públicas, de organismos de derecho

17 La provisión de liquidez de urgencia fue utilizada como modalidad de asistencia financiera prestada el 25 de junio de 2012 a Chipre. Al respecto, véase la Decisión del Consejo de Gobierno del BCE relativa a la provisión de liquidez de urgencia a solicitud del Banco Central de Chipre; declaraciones del Eurogrupo de 25 de marzo, de 12 de abril, de 13 de mayo y de 13 de septiembre sobre Chipre; la Decisión del Consejo (2013/236/UE), de 25 de abril de 2013, dirigida a Chipre y relativa a las medidas específicas destinadas a restablecer la estabilidad financiera y un crecimiento sostenible (D.O.U.E. L141/32, de 28.5.2013); y el Memorándum de Entendimiento de 26 de abril de 2013 sobre Condiciones Específicas de Política Económica, celebrado entre Chipre y el Mecanismo Europeo de Estabilidad.

El 17 de mayo de 2017, el BCE aprobó el Acuerdo de asistencia de liquidez de emergencia, que incorpora su definición y condiciones. Disponible en: https://bit.ly/2TwTJhj.

No obstante, su carácter de instrumentos financieros resulta discutida por la doctrina. Así, Ruffert (2011: 1788) considera que no todos los bonos adquiridos por el BCE tienen la misma naturaleza, y muchos no son «instrumentos financieros». Por el contrario, los estiman, sin distinción, «instrumentos financieros» De Grauwe (2012) y Smits (2012). 
público o empresas públicas de los Estados miembros (art. 123.1 TFUE), aunque algunos miembros del propio Consejo de Gobierno del BCE y, en particular, el TCFA ha planteado, como se verá a continuación, ciertas dudas acerca de si la compra de bonos soberanos en los mercados secundarios constituye una forma encubierta de eludir esta prohibición.

El primero de los programas de compra de bonos soberanos en los mercados primarios y secundarios puesto en marcha, con carácter extraordinario y temporal, por el BCE, tuvo lugar en 2009, bajo la modalidad de «bonos garantizados», de conformidad con la Decisión de 2 de julio de 2009, que tenía su fundamento jurídico en el art. 105, 2 primer guion TFUE y los arts. 12.1, en relación con el primer guion del art. 3.1, y el 18.1 de los Estatutos del SEBC, aplicándose a Grecia, Irlanda y Portugal hasta su expiración el 30 de junio de $2010^{19}$.

Esta práctica del BCE fue cuestionada, junto a otras actuaciones de la Unión Europea para afrontar la crisis económico-financiera, en el recurso planteado ante el TCFA respecto a la asistencia financiera a Grecia (Sentencia de 7 de septiembre de 2011) ${ }^{20}$, desde la perspectiva de la necesidad de garantizar la intervención del Bundestag en los instrumentos intergubernamentales creados por los Estados de la eurozona para hacer frente a la crisis económico-financiera. En su pronunciamiento, el TCFA consideró, de modo muy genérico, que la prohibición de la compra directa de instrumentos de deuda de las instituciones públicas por el BCE también contribuye a salvaguardar los requisitos constitucionales de la democracia en la Unión (para. 129).

Ese mismo año, el BCE adoptó el programa de bonos en el mercado de valores (Securities Markets Programme, SMP por sus siglas en inglés), en cuyo marco fue aprobado, el 3 de noviembre de 2011, el segundo programa de adquisiciones de «bonos garantizados», aplicado a Italia y España ${ }^{21}$, junto a una informal (y reservada), pero contundente, presión del BCE sobre los referidos Estados miembros para adoptar, en contrapartida, reformas estructurales en su política económica.

19 Decisión del Banco Central Europeo, de 2 de julio de 2009 (2009/522/CE), sobre la ejecución del programa de adquisiciones de instrumentos de renta fija tipo Pfandbrief (bonos garantizados) (DOUE L 175/18, de 4.7.2009).

20 Resolución de 7 de septiembre de 2011. BVerfG, decisión del Segundo Senado (2 BvR 987/10), disponible en inglés en: https://bit.ly/37GslpF.

21 Decisión del Banco Central Europeo, de 14 de mayo de 2010 (BCE/281/UE), por la que se crea el programa para mercados de valores (DOUE L 124/8, de 20.5.2010) y Decisión del Banco Central Europeo, de 3 de noviembre de 2011 (2011/744/UE), sobre la ejecución del segundo programa de adquisiciones de bonos garantizados (DOUE L 297/70, de 16.11.2011). 
Un apremio, ejercido para vincular la compra de bonos soberanos al cumplimiento de una condicionalidad económico-financiera que se puso en evidencia, en el caso italiano, como consecuencia de la publicación por un conocido medio de la prensa escrita de la carta remitida al primer ministro italiano por M. Draghi y J.-C. Trichet, instando al Gobierno italiano a adoptar, lo antes posible, «mediante decretos-leyes», detalladas reformas económicas y reformas estructurales y legales ${ }^{22}$. Una carta similar, que no fue hecha pública, fue enviada al presidente del Gobierno español, según consta en la Decisión del Defensor del Pueblo Europeo por la que se archiva la investigación sobre la reclamación 2016/2011/AN contra el Banco Central Europeo ${ }^{23}$.

El 6 de septiembre de 2012 el Consejo de Gobierno del BCE adoptó el programa denominado "Características técnicas de las operaciones monetarias de compraventa de títulos en el sistema euro" (Outright Monetary Transactions, OMT, por sus siglas en inglés $)^{24}$, por el que se daba por concluido el programa SMP y se implantaba un nuevo programa de compra de bonos soberanos en los mercados secundarios.

En el contexto de un recurso presentado ante el TCFA contra la reforma del art. 136 TFUE, cuestionando también la legislación alemana que ratifica el Tratado MEDE y la que autoriza su participación en el mismo, junto a la ratificación del TECG, en la Sentencia de 12 de septiembre de $2012^{25}$, el Alto Tribunal alemán declaró que estaría prohibida la adquisición de bonos soberanos en el mercado secundario por el BCE si esta se realizara con la «intención» de financiar las economías de los Estados miembros al margen de los mercados de capitales (para. 174).

Aun cuando hasta entonces el TCFA se había venido pronunciando obiter dicta respecto de la compra de bonos soberanos en los mercados secundarios por el BCE, el programa de compra OMT adoptado en 2012 fue objeto de un recurso específico ante el Alto Tribunal alemán con los argumentos del riesgo que entrañaba para los contribuyentes alemanes ${ }^{26}$ y el menoscabo que suponía del poder presupuestario del Bundestag.

22 Originariamente, la carta fue publicada por el diario Corriere della Sera y, a partir de esa publicación, en toda la prensa europea. En España, el texto íntegro en español fue publicado por El Pais. Disponible en: https://bit.ly/33xLdo3.

23 Disponible en: https://bit.ly/39f2N3F.

24 Disponible en: https://bit.ly/37J055S.

25 Resolución de 12 de septiembre de 2012. BVerfG, decisión del Segundo Senado (2 BvR 1390/12), disponible en inglés en: https://bit.ly/2HyAiSI.

26 Desde el punto de vista fiscal, Wolff (2013: 30) estima que el programa OMT no tiene unas implicaciones fiscales distintas a las consecuencias de cualquier otra política monetaria. 
En este caso, el TCFA decidió plantear al TJUE dos cuestiones prejudiciales que ponían en cuestión la legalidad del programa de compra de bonos soberanos OMT: la primera de ellas por la posible conculcación del art. 123 TFUE, y, la segunda, por exceder el mandato del BCE al incidir en el ámbito de la política económica (ultra vires) ${ }^{27}$.

Abordando como objeto de su pronunciamiento, ahora sí, el análisis de un concreto programa de compra de bonos soberanos en mercados secundarios como el OMT, que vincula la condicionalidad del Estado miembro a la Facilidad Europea de Estabilización Financiera (FEEF) y, posteriormente, al MEDE, el TCFA debía manifestar su criterio, mediante la estricta aplicación del derecho de la Unión y los tratados intergubernamentales adoptados por los Estados miembros de la eurozona para prestar asistencia financiera a otros Estados de la zona euro que la soliciten.

Ciertamente, como señalan Jędrzejowska-Schiffauer y Schiffauer (2016: 201), tanto la Facilidad Europea de Estabilización Financiera (FEEF) como el MEDE, constituyen instrumentos de política económica, pero, en su opinión, el hecho de que el BCE establezca la condicionalidad de la compra de bonos soberanos a través de la aplicación de dichos programas de asistencia financiera «no significa» que el BCE rebase por ello, automáticamente, el ámbito de su política monetaria.

En esta línea interpretativa cabe situar la resolución de dichas cuestiones prejudiciales por el Tribunal de Justicia de la UE a través de la Sentencia de 16 de junio de 2015, asunto C-62/14, Gauweiler, estimando, en primer lugar, que el TFUE y los Estatutos del SEBC, deben interpretarse en el sentido de que "autorizan» al BCE a adoptar un programa de compra de bonos soberanos en los mercados secundarios como el OMT (para. 127), siendo procedente reconocerle una «amplia facultad de apreciación» dado el carácter técnico y complejo de sus decisiones (para. 68) ${ }^{28}$.

También estima que dicho programa no «viola el principio de proporcionalidad» (para. 99), y, en última instancia, que, al incorporar como condición para los Estados miembros beneficiarios el compromiso de aplicar un programa

De hecho, con carácter general, Simon (2015: 2019) afirma que "prácticamente todas» las actuaciones monetarias del BCE tienen efectos económicos y consecuencias en la política económica de los Estados miembros.

27 Resoluciónde14deenerode2014.BVerfG,decisióndelSegundoSenado(2BvR2728/13), https://www.bundesverfassungsgericht.de/SharedDocs/Entscheidungen/EN/2014/ 01/rs20140114_2bvr272813en.html. Véase al respecto Caponi (2014: 469-478).

28 A juicio de Jędrzejowska-Schiffauer y Schiffauer (2016: 201), la «justificación última» de esta discrecionalidad se encuentra en la «imposibilidad metodológica» de trazar una línea divisoria entre la política económica y la monetaria. 
de ajuste estructural, la emisión del programa OMT no está prohibida por el art. 123.1 TFUE (para. 121).

De este modo, el asunto Gauweiler no solo aborda importantes cuestiones que afectan a las relaciones entre el ordenamiento de la Unión y los ordenamientos nacionales, sino que, específicamente, tiene «implicaciones cruciales para la arquitectura de la gobernanza económica europea» (Fabbrini, 2016: 6).

No obstante, la polémica relativa a los programas de compra de bonos soberanos en mercados secundarios tardó poco tiempo en reabrirse, pues unos meses antes del pronunciamiento del Tribunal de Justicia en el asunto Gauweiler, el 4 de marzo de 2015, con base en el art. 127.2, primer guion TFUE y el art. 12.1, párrafo segundo, art. 3.1, primer guion, y art. 18.1 de los Estatutos del SEBC, el BCE adoptó el programa de compras de valores públicos en mercados secundarios (Public Sector Purchase Programme, conocido como PSPP ${ }^{29}$, puesto en marcha el 9 de marzo de 2015.

De nuevo, el TCFA planteó al TJUE cinco cuestiones prejudiciales cuestionando la conformidad del programa PSPP de compra de bonos soberanos en los mercados secundarios por el $\mathrm{BCE}$ con el ordenamiento $\mathrm{UE}^{30}$; en concreto, dos de los argumentos principales habían sido planteados en el asunto Gauweiler: la posible vulneración del art. 123 TFUE, la hipotética extralimitación del mandato del BCE al incidir en la política económica (ultra vires), de modo que el Alto Tribunal alemán solicita ahora la concreción de ciertos aspectos que dicha sentencia no había aclarado completamente con anterioridad.

Resolviendo las cuestiones prejudiciales del TCFA mediante la Sentencia de 11 de diciembre de 2018, asunto C-493/17, Weiss, el TJUE se pronuncia sobre una serie de cuestiones concretas relativas a los programas de compra de bonos soberanos en los mercados secundarios que no habían sido precisadas en el asunto Gauweiler: la motivación de los actos de la Unión; el concepto de política monetaria en el ordenamiento de la Unión; la proporcionalidad con los objetivos de política monetaria del programa de compra de bonos soberanos, y, en última instancia, su compatibilidad de facto con el art. 123.1 TFUE.

En primer término, el TJUE estima que la motivación de los actos de la Unión no exige que se especifiquen todas las razones «de hecho o de Derecho» pertinentes, $y$, en particular, que no puede exigirse una motivación específica

29 Decisión (UE) 2015/774 del Banco Central Europeo, de 4 de marzo de 2015, sobre un programa de compras de valores públicos en mercados secundarios (DOUE L 121/20, de 14.5.2015), modificada por la Decisión (UE) 2017/100 del Banco Central Europeo, de 11 de enero de 2017 (DOUE L 16/51, de 20.1.2017).

30 Resolución de 18 de julio de 2017, decisión del Segundo Senado (2 BvR 859/15), disponible en inglés en: https://bit.ly/3mpdQdT. 
«para cada una de las decisiones técnicas» adoptadas en el cumplimiento de los objetivos de la Unión. Además, la valoración de la suficiencia de la motivación debe tomar en consideración no solo «el tenor del acto", sino también «su contexto» y «el conjunto de normas jurídicas» reguladoras de la materia (paras. 31-33), concluyendo que la documentación aportada por el BCE sobre el programa PSPP incluye justificaciones suficientes, incluidas informaciones públicadas en prensa (paras. 35 a 37), lo que permite concluir que, en consecuencia, la Decisión del BCE no es inválida (para. 44).

En segundo término, considera que corresponde al BCE el cumplimiento de los objetivos de la política monetaria — cuyo concepto, según afirma, no está definido en los tratados, que plantean, simplemente, el cumplimiento de objetivos (para. 50) —, sin vulnerar las limitaciones impuestas en los tratados mismos, en especial, el principio de atribución, cuyo cumplimiento es controlado por el TJUE (paras. 48-49).

De este modo, teniendo en cuenta el objetivo perseguido por el programa de compra de bonos PSPP y los medios previstos para alcanzarlo, el TJUE entiende que este se sitúa en el ámbito de la política monetaria (para. 70), sin que sea óbice para alcanzar esta consideración los efectos indirectos desplegados por dicha medida, para lo que se remite a lo declarado en las sentencias Pringle y Gauweiler (para. 63).

En tercer lugar, el TJUE reconoce que la proporcionalidad del programa PSPP se define a partir del concepto general de proporcionalidad de los actos de las instituciones de la Unión establecido en su propia jurisprudencia, es decir, atendiendo a la idoneidad de los instrumentos adoptados para alcanzar los fines y al respeto de los límites de lo que sea necesario para alcanzarlos (para. 72).

Esta valoración del respeto del principio de proporcionalidad analiza también la toma en consideración por el $\mathrm{BCE}$ de los riesgos que el programa PSPP entrañaba para los bancos centrales de los Estados miembros (duración predeterminada del programa, restricciones establecidas al volumen de compra, y control de los riesgos de posibles pérdidas), concluyendo que el programa de compra de bonos soberanos PSPP no vulnera el principio de proporcionalidad (para. 100).

En última instancia, el Tribunal de Justicia retoma el argumento ya planteado en la sentencia Gauweiler sobre la conformidad de las operaciones de compra de bonos soberanos en los mercados secundarios con las prescripciones contempladas en el art. 123.1 TFUE. A este respecto, considera que el programa PSPP contiene garantías suficientes para prevenir la previsibilidad de la compra en los mercados, y, de este modo, garantizar que solo constituye un incentivo para que los Estados miembros mantengan un presupuesto sólido (para. 135-136), en tanto que la principal fuente de financiación de los mismos sigue siendo el mercado (para. 141). A resultas de estas apreciaciones, el TJUE estima que el programa PSPP respeta tanto las previsiones del art. 123 TFUE como del art. 18.1 de los Estatutos del SEBC (para. 157). 
En definitiva, a grandes rasgos, la sentencia Weiss no aporta grandes novedades respecto de lo ya afirmado en el asunto Gauweiler sobre los programas del BCE de compra de bonos soberanos en mercados secundarios. Sin embargo, partiendo de esta última, el TJUE lleva a cabo precisiones importantes respecto de la imbricación entre la política monetaria y la política económica y, en sentido contrario, los criterios susceptibles de mantener la diferenciación entre ellas.

Así, de acuerdo con los tratados, el objetivo perseguido se convierte en el elemento definidor del carácter monetario o económico de la medida o intervención adoptada (Mooij, 2019: 463), sin que los efectos indirectos puedan tener la capacidad de alterar esta naturaleza, de tal modo que las funciones del BCE no parecen haber cambiado de modo significativo. Sin embargo, vistas en perspectiva, la sucesión de pronunciamientos del Tribunal de Justicia en los asuntos Pringle, Gauweiler y Weiss ofrece el retrato de una construcción pretoriana de la Unión Económica y Monetaria Europea, o, cuando menos, la constatación de las deficiencias con las que esta fue alumbrada.

En efecto, frente a la utópica consideración estanca de la Unión Económica y la Unión Monetaria, la realidad se ha impuesto evidenciando que una unión monetaria «afecta profundamente» al acervo jurídico-político de los Estados miembros involucrados (Hahn, 1991: 784).

\section{LA SENTENCIA DEL TRIBUNAL CONSTITUCIONAL FEDERAL ALEMÁN DE 5 DE MAYO DE 2020: DE LAS PALABRAS A LOS HECHOS}

El 5 de mayo de 2020 el Tribunal Constitucional Federal alemán ha transformado las amenazas y avisos que llevaba años enviando al TJUE en una sentencia en la que persigue actuar como el guardián de la integración europea, vigilando sobre las conductas ultra vires de sus instituciones ${ }^{31} \mathrm{y}$, de alguna manera, defender la esencia de la Constitución alemana. En dicha sentencia, el TCFA básicamente suspendía la vinculatoriedad para Alemania de la citada decisión del mismo tribunal europeo de diciembre de 2018 (para. 163), a no ser que se integrase la justificación del programa PSPP y se motivase la proporcionalidad de las medidas en un plazo de tres meses.

Desde hace décadas, el TCFA ha sembrado las semillas de los argumentos que se encuentran en esta sentencia reciente, afirmando que se abstendría de ejercer jurisdicción sobre las actuaciones de las instituciones europeas, y de hecho sobre las normas de derecho comunitario a condición de que (solange)

31 BVerfG, 2 BvR 859/15, 2 BvR 980/16, 2 BvR 2006/15, 2 BvR 1651/15, decisión del Segundo Senado del 5 de mayo de 2020, disponible en: https://bit.ly/368TyAh. 
las mismas garantizaran un nivel de protección de los derechos compatible con la Constitución alemana ${ }^{32}$ y no hubiese extralimitaciones (Grimm, 2020: 944; Wendel, 2020: 979).

En las decisiones relativas a los Tratados de Maastricht y de Lisboa, ya se aclararon temas esenciales para comprender la evolución de la jurisprudencia del TCFA ${ }^{33}$. En particular, se examinaba el alcance inviolable del principio democrático (según la perspectiva alemana) y se afirmaba la misión del mismo tribunal de verificar que las actuaciones de las instituciones europeas se desenvolvieran dentro de las competencias asignadas. Así, el mismo TCFA «erigía una muralla a la progresión de la integración que alertaba de lo que iba a hacer: su oportunidad en mayo de 2020» (Mangas Martín, 2020: 4), precedida por las dudas que planteó en las cuestiones prejudiciales con respecto a las medidas adoptadas por el BCE a raíz de la crisis económica. Por todo ello, los contenidos de la sentencia que aquí se comenta estaban anticipadas en las decisiones anteriores, también con respecto al OMT ${ }^{34}$.

Desde una perspectiva sustancial, lo que el TCFA hace es averiguar la validez de las normas europeas en su relación con las normas constitucionales nacionales. Sin embargo, basa desde siempre su razonamiento en el control del respeto, por parte de los órganos políticos alemanes, de sus obligaciones constitucionales internas: el hecho de que se involucren en el juicio normas europeas sería, así, un mero accidente.

Es interesante que Italia hubiese solicitado en 2017 que la cuestión prejudicial fuese inadmitida de plano, alegando que el TCFA intentaba así entrar a juzgar la validez de un acto de la UE desvirtuando la naturaleza de la cuestión prejudicial; sostenía, además, que el mismo tribunal no reconocía valor vinculante a la futura decisión del TJUE, erigiéndose juez último de la interpretación y validez de las normas europeas conforme a los estándares alemanes. El TJUE desestimó esta solicitud recordando, por un lado, que sus decisiones son vinculantes para el juez nacional que las solicita y admitiendo, por otro lado, que cabe la posibilidad de una cuestión prejudicial planteada en un caso en el que de manera incidental se pone en duda la validez de un acto de la UE.

32 Se trata de las dos sentencias conocidas como Solange I, 2 BvL 52/71, de 29 de mayo de 1974 (BVerfGE 37, 271) y Solange II, 2 BvR 197/83, de 22 de octubre de 1986 (BVerfGE 73, 339).

33 Para el análisis de la evolución hasta 2010 de la jurisprudencia con referencia a la protección de derechos, véase González Pascual (2010: 124-146).

34 Para una reconstrucción completa, véase también Sáinz de Vicuña y Barroso (2016: 1067). 


\section{LOS ARGUMENTOS DEL TCFA SOBRE FALTA DE MOTIVACIÓN Y PROPORCIONALIDAD}

En primer lugar, como se acaba de ver, el TCFA lamentaba que las medidas relacionadas con el PSPP carecían de una motivación específica con respecto a necesidad, entidad y duración en su relación con la política monetaria y los efectos para la política económica. Considera ahora que la respuesta del TJUE es insuficiente a su vez, ya que no proporciona un análisis detallado de la motivación misma, ni argumenta correctamente al respecto ${ }^{35}$.

En segundo lugar, creía que el BCE no tenía una competencia específica para adoptar aquellas medidas y que no había justificado por qué no estaba actuando con instrumentos coherentes con su mandato monetario (principal), de mantenimiento de la estabilidad de los precios, en lugar de emplear instrumentos que invadían la competencia económica y fiscal de los Estados miembros, situándose así ultra vires ${ }^{36}$. La decisión del TJUE, en opinión del TCFA, no proporciona argumentos convincentes y, además, los jueces europeos habrían renunciado a desempeñar su rol de guardianes de los tratados, consintiendo una expansión ilegítima de los poderes del BCE (paras. 162 y 163). Con referencia al principio de proporcionalidad conforme al art. 5 TUE, el TCFA critica la ausencia en la sentencia del TJUE de un análisis de los efectos económicos y le reprocha su autolimitación al examen de los excesos manifiestos por parte del BCE y de posibles efectos adversos también manifiestamente desproporcionados con respecto al objetivo perseguido.

La ponderación equivocada realizada por el BCE se quedaría así exenta de control jurisdiccional, y ello para el TCFA resulta inaceptable. Finalmente, además de contestar la actuación del BCE, como resulta evidente en los argumentos que se han brevemente resumido, el TCFA critica duramente al TJUE, acusándole también de conductas ultra vires, pues define partes de la sentencia de 2018 como incomprensibles, arbitrarias y básicamente extrañas a su mandato judicial.

\section{2. ¿UUN CORTOCIRCUITO NACIONAL? CIUDADANOS Y TCFA VS. INSTITUCIONES DEMOCRÁTICAS}

Un elemento de interés de este caso está representado por el cortocircuito democrático que supone a nivel interno, dentro de la misma Alemania,

35 Críticos sobre esta parte de la sentencia del TJUE, Mangas Martín (2020: 8) y Galetta y Ziller (2020: 179).

36 Una reconstrucción crítica al respecto puede encontrarse en Pace (2020: 299); Schneider (2020: 968) intenta individualizar los aspectos positivos de dicha doctrina. 
en el que se ha creado una suerte de conflicto entre dos bandos, es decir, entre ciudadanos apoyados por el TCFA y ejecutivo y legislativo federales.

En efecto, lo que ha desencadenado los hechos fueron los recursos de amparo (Verfassungsbeschwerde), planteados en Karlsruhe por Heinrich Weiss y otros, entre ellos, Bernd Lucke (uno de los fundadores del partido Alternative für Deutschland), y el miembro de la CSU (Christlich-Soziale Union) bávara Peter Gauweiler, con el apoyo del profesor Markus Kerber. El objetivo fundamental era evitar que se llegase a compartir los riesgos dentro del sistema UE, afectándose así los equilibrios entre ganancias y pérdidas de los bancos centrales y en consecuencia vulnerando la identidad constitucional alemana conforme al art. 79.3 de la Constitución (que protege el núcleo duro de la misma). Según ellos, si los efectos económicos de recapitalización de los bancos centrales hubiesen sido de tal envergadura, habría sido necesaria la involucración del Bundestag; órgano, este, que no se había opuesto a la compra de bonos hasta el momento (ni lo había hecho el Gobierno federal).

$\mathrm{Si}$ bien el TCFA no sancionaba en mayo la inercia de los órganos políticos nacionales, pues no llegó a declarar su responsabilidad por omisión, los consideraba empero obligados a oponerse al PSPP en su forma actual. Les ordenaba que, junto con el Banco Central alemán (Bundesbank), consiguieran por parte del BCE una decisión que justificase, de forma comprensible y comprobada, que los fines de política monetaria que el PSPP pretendía alcanzar no iban a tener efectos desproporcionados por lo que atañe a las políticas económica y fiscal. En caso contrario, el mismo Bundesbank no estaría facultado para tomar parte en la aplicación del sistema, no pudiendo ya adquirir bonos y estando obligado a vender aquellos que ya posea. En otras palabras, puesto que a ninguna autoridad alemana se le puede exigir implementar o ejecutar actos europeos ultra vires, transcurrido el plazo de tres meses que el TCFA estimaba suficiente para una coordinación del Eurosistema, las obligaciones de participación en el PSPP habrían caducado. Un verdadero ultimátum establecido por el TCFA para las instituciones europeas (Tesauro y De Pasquale, 2020: 14).

\section{REACCIONES INSTITUCIONALES Y CRÍTICAS DOCTRINALES}

En términos teóricos y absolutamente generales, es de apreciar que el TCFA en los casos analizados haya planteado cuestiones prejudiciales, sin proceder a interpretar de manera unilateral las normas de derecho de la UE (Sáinz de Vicuña y Barroso 2016: 1095), lo cual demuestra una parcial superación de la actitud totalmente reacia al diálogo que en el pasado había mantenido (Ragone, 2011). Y, para individualizar una vertiente positiva del asunto, quizá su intervención pueda servir para reabrir el debate político sobre una capacidad fiscal de la UE que refuerce y legitime las actuaciones del BCE de los últimos años (Cafaro, 2020), aunque no parece muy probable. 
Sin embargo, la sentencia del TCFA presenta una serie de problemas metodológicos, desde una perspectiva jurídica, y otros de oportunidad, desde una perspectiva política.

Por un lado, y por muy obvio que pueda parecer, si bien la decisión del TJUE de diciembre de 2018 tenía una motivación deficiente, ninguna norma permite no acatarla por estar mal escrita. Además, como señaló el abogado general en sus conclusiones sobre el caso Gauweiler, si se reconociese a cualquier tribunal constitucional nacional la posibilidad de seleccionar las sentencias europeas vinculantes, se rompería el pacto constitucional que subyace a la construcción europea, que tiene entre sus fundamentos la cesión al TJUE de la "competencia sobre las competencias» (Kompetenz-Kompetenz). Así, la sentencia quebraría «la primacía del derecho de la UE, la unidad del ordenamiento común y la seguridad jurídica», al cuestionar la autoridad del órgano jurisdiccional a cargo de la interpretación y aplicación de los tratados (Mangas Martín, 2020: 11). Al mismo tiempo representaría un peligroso precedente para tribunales y gobiernos críticos con la integración europea y proclives a argumentos «nacionalistas», como es el caso de Polonia y Hungría (Caravita et al., 2020: 12).

Si se tiene en cuenta el parámetro empleado en el juicio, al declarar la «inconstitucionalidad» (en parte) del programa PSPP, el TCFA estaría vulnerando el derecho de la UE y la seguridad jurídica (Mangas Martín, 2020: 7-8) y más en general el principio básico del derecho internacional, pacta sunt servanda (Tesauro y De Pasquale, 2020: 18).

Adicionalmente, hay que tener en cuenta que la valoración del TCFA se basa en una "entelequia", es decir, la distinción entre política monetaria y política económica (Lozano Cutanda, 2020: 3; en un sentido parecido, Jiménez-Blanco y Carrillo de Albornoz, 2020), y cae en una contradicción lógica; ello porque considera ilegítimo el programa PSPP por falta de ponderación y proporcionalidad en las evaluaciones del BCE. Sin embargo, el TCFA no puede a priori asegurar que esta ponderación no haya tenido lugar; solo puede constatar que no es posible averiguarlo. Según la doctrina, este razonamiento esconde la verdadera finalidad de la sentencia (Pace, 2020: 307).

Las mismas indicaciones que el TCFA da al BCE parecen ser una extralimitación del primero (Ciancio, 2020: 35) al configurarse como una «instrucción» que viola la independencia del segundo conforme al art. $130 \mathrm{TFUE}^{37}$. El

37 Textualmente: «[...] en el ejercicio de las facultades y en el desempeño de las funciones y obligaciones que les asignan los Tratados y los Estatutos del Sistema Europeo de Bancos Centrales y del Banco Central Europeo, ni el Banco Central Europeo, ni los bancos centrales nacionales, ni ninguno de los miembros de sus órganos rectores podrán solicitar o aceptar instrucciones de las instituciones, órganos u organismos de la Unión, ni de los Gobiernos de los Estados miembros, ni de ningún otro órgano. Las instituciones, órganos u organismos de la Unión, así como los Gobiernos de los 
TCFA manifiesta una actitud opuesta a la del TJUE, que en la sentencia Weiss había reconocido un margen amplio de apreciación para el Sistema Europeo de Bancos Centrales y se había centrado en averiguar si las decisiones del BCE estaban motivadas y no presentaban errores manifiestos de apreciación. El TJUE confiaba así en la diligencia y precisión del BCE a la hora de elegir e implementar los medios técnicos necesarios.

Políticamente, la sentencia del TCFA ha sido atacada, como mínimo, por su «inoportunidad» en una fase tan delicada y decisiva de la integración europea y de los equilibrios entre los Estados (Caravita et al., 2020: iv; menos críticos Rovetta y Smiatacz, 2020: 435). Con argumentos similares, se ha criticado que el TCFA, tras reprocharle al BCE haber actuado sin tener en cuenta los efectos sobre la política económica, adoptase una sentencia que, aun cuando se pudiese considerar parte de sus competencias (lo cual es cuestionable), «no ha medido adecuadamente sus efectos colaterales económicos, jurídicos y políticos en un momento especialmente crítico» (Lozano Cutanda, 2020: 6). En términos generales, la decisión del TCFA se superpone y opone al resultado de meses de negociaciones y actuaciones diplomáticas y en el seno de las instituciones europeas, transfiriendo a nivel de la UE una visión puramente alemana de la proporcionalidad, sin realizar una ponderación adecuada de los intereses en juego ni de los efectos positivos (Poiares Maduro, 2020).

Por todo ello, las reacciones de las instituciones europeas no se han hecho esperar.

El mismo día, el BCE difundió un comunicado de prensa en el que, de manera sintética pero concluyente, revalidaba su compromiso para hacer todo lo necesario, dentro de su mandato, para asegurar que la inflación siguiera situándose en un nivel coherente con sus fines a medio plazo, y recordaba que el TJUE ya había confirmado que estaba actuando dentro de su competencia relativa a la estabilidad de precios en la sentencia de 2018. La presidenta Lagarde añadía que los referentes principales serían, en todo caso, el Parlamento Europeo y el TJUE.

Tres días más tarde, el TJUE a su vez hizo público un comunicado de prensa bastante insólito ${ }^{38}$, en el que, tras reiterar que nunca hace comentarios acerca de las decisiones adoptadas a nivel estatal, recordaba (al TCFA) que sus sentencias dictadas con carácter prejudicial son vinculantes, para el órgano judicial nacional, en la resolución del litigio principal; que el derecho de la UE tiene primacía sobre los derechos nacionales y que solo el mismo TJUE puede

Estados miembros, se comprometen a respetar este principio y a no tratar de influir en los miembros de los órganos rectores del Banco Central Europeo y de los bancos centrales nacionales en el ejercicio de sus funciones».

38 Tribunal de Justicia de la UE, comunicado de prensa n. 58/20, Luxemburgo, 8 de mayo de 2020, disponible en: https://bit.ly/3jw5tLQ. 
juzgar la validez de los actos europeos. Justificaba su intervención por haber recibido numerosas solicitudes de aclaraciones después de la sentencia alemana y anticipaba que no difundiría comunicaciones posteriores al respecto.

El mismo TJUE habría sido competente si la Comisión hubiese decidido abrir un procedimiento de infracción, conforme al art. 258 TFUE, en contra de Alemania (opción que la misma presidenta afirmó no descartar por completo el 10 de mayo $\left.^{39}\right)$. En efecto, es posible que el incumplimiento por parte de un Estado sea el resultado de la actuación de un órgano jurisdiccional, además de la consecuencia de la conducta de instituciones políticas nacionales. Existe en este sentido un reciente precedente en el que el órgano interesado era el Consejo de Estado francés ${ }^{40}$. De momento, al cabo de tres meses, la Comisión no ha terminado su análisis de la sentencia de mayo que podría emplearse para abrir el expediente de infracción.

¿Bien está lo que (por ahora) bien acaba? Desde mayo, se han sucedido actuaciones de distintas instituciones para desactivar los efectos de la sentencia que podrían poner en entredicho las medidas ya en pie y las futuras medidas del BCE para responder también a la crisis económica generada por la pandemia por COVID-19. A los pocos días, la canciller Angela Merkel había promovido nuevas negociaciones dirigidas a alcanzar una decisión sobre medidas parecidas y el ministro de Finanzas había declarado que, según su criterio, el Bundesbank podría seguir participando en las compras del BCE.

El presidente del Bundesbank solicitó al BCE la autorización para transmitir al ejecutivo y al legislativo alemanes algunos documentos confidenciales que probarían la ponderación por parte del BCE mismo de su intervención en los mercados, sin incurrir en aquellas extralimitaciones evocadas por el TCFA. Esta actuación, entre otras, ha favorecido que el Bundestag pudiera aprobar, el 2 de julio, una resolución que daba por cumplida la petición del 5 de mayo, afirmando que está comprobado que el BCE había sopesado todas las consecuencias del programa PSPP llegando a valorar que el impacto positivo en la política monetaria justificaría su realización. El mismo presidente del Bundesbank, en nombre del consejo directivo, aclaró que se pueden considerar satisfechas las exigencias establecidas por el TCFA, sumándose a las opiniones del Bundestag y del Gobierno federal.

Empero, la historia no se ha acabado aquí. Los recurrentes han solicitado que el TCFA averigüe el cumplimiento fehaciente de la sentencia de 2020 y ello podría llevar a una nueva revisión de los datos y documentos facilitados

39 Declaración de la Presidenta de la Comisión Europea, de 10 de mayo de 2020, disponible en: https://bit.ly/2Tn0tyu.

40 Sentencia del 4 de octubre de 2018, asunto C-416/17, Comisión c. Francia. 
por el BCE (y en consecuencia a una nueva afirmación de la obligación del Bundesbank de salir del programa $)^{41}$.

\section{IMPACTO SOBRE LA RESPUESTA A LA PANDEMIA POR COVID-19 Y RELEVANCIA PARA LOS CONFLICTOS ENTRE ESTADOS Y ORGANIZACIONES INTERNACIONALES MÁS ALLÁ DE EUROPA}

A la hora de dictar la sentencia del 5 de mayo y de darla a conocer, el propio TCFA en su comunicado de prensa afirmó que la misma no afectaba a «ninguna de las medidas de asistencia financiera adoptadas por la Unión Europea o el Banco Central Europeo en el contexto de la crisis del coronavirus».

Sin embargo, aunque aclarase que el razonamiento se circunscribía al programa PSPP y no al programa excepcional para hacer frente a la pandemia por COVID-19, el TCFA estaba dando unas cuantas pautas al BCE sobre lo que considera equilibrado, estableciendo una serie de condiciones necesarias para que no se estime vulnerada la prohibición de financiación monetaria de los Estados miembros. Entre otras: el volumen limitado de compras desde el inicio del programa; la publicación de información agregada sobre las características de las compras; el límite, para los bancos centrales del Eurosistema, del 33\% de una determinada emisión de bonos de una administración de un Estado miembro o del 33\% de los valores en circulación de uno de los Estados; la proporción de los volúmenes de compras en comparación con la proporción de capital; las limitaciones a la compra de valores de entidades públicas solo si el cedente está en posesión de una certificación de calidad crediticia mínima que permita acceso al mercado; el carácter siempre limitado y discontinuo de las compras que deben terminar cuando ya no sean necesarias para alcanzar el objetivo de estabilizar la inflación al $2 \%$.

En consecuencia, como efecto colateral, voluntariamente deseado o no, el hecho es que la sentencia del TCFA puede suponer una hipoteca para los programas que se implementen con el fin de afrontar las consecuencias económicas derivadas de la pandemia por COVID-19 (Faraguna, 2020: 309). No parece muy relevante que se trate de una «respuesta a un problema coyuntural, pasajero" (Roca, 2020: 2849), como es el caso del programa de compras de emergencia (Pandemic Emergency Purchase Programm, PEPP) recientemente activado por el $\mathrm{BCE}$, en unas circunstancias en las que, como ha reconocido

41 El debate sigue abierto en los foros políticos y mediáticos, como lo ejemplifica el editorial de El País del 8 de agosto: Defensa del BCE. Bruselas debe actuar ya contra el Tribunal Constitucional alemán, https://elpais.com/opinion/2020-08-07/defensa-del-bce.html. 
Kristalina Georgieva (directora gerente del Fondo Monetario Internacional, FMI), «los costes humanos de la pandemia del Coronavirus ya son inconmensurables y todos los países necesitan trabajar juntos para proteger a las personas y limitar el daño económico. Este es un momento de solidaridad [...]» ${ }^{42}$.

Adicionalmente, el impacto de la sentencia de Karlsruhe, a diferencia de Solange, va dirigido directamente contra el derecho originario, donde se afirma que el TJUE «garantizará el respeto del Derecho en la interpretación y aplicación de los Tratados» (art. 19.1 TUE). Ello implica, como resulta conocido, que no es su único intérprete en tanto que comparte dicha función con los órganos jurisdiccionales nacionales, pero sí su intérprete supremo, al que quedan vinculados aquellos. De hecho, a través de la cuestión prejudicial prevista en el actual art. 267 TFUE — exportada por el Protocolo de Tegucigalpa de 13 de diciembre de 1991, a la Corte Centroamericana de Justicia, y por el Protocolo de Cochabamba de 1996, a la Corte de Justicia de la Comunidad Andina-, el TJUE está llamado a pronunciarse sobre la «interpretación de los Tratados», y sobre la «validez e interpretación de los actos adoptados por las instituciones, órganos u organismos de la Unión", conformando un auténtico modelo de diálogo entre tribunales (De Vergottini, 2010). Un diálogo en ocasiones conflictual, pero que nunca hasta hoy había supuesto la suplantación, por la vía de los hechos, de las funciones que competen al TJUE en relación con el derecho de la UE por un tribunal constitucional nacional.

En última instancia, la sentencia del TCFA de 5 de mayo representa un ulterior ejemplo de la tendencia a desafiar la autoridad de órganos jurisdiccionales supra e internacionales que se ha dado tanto en Europa como en América Latina, devolviendo el impulso al antiguo debate sobre la supervivencia de la soberanía nacional en los sistemas de integración supranacional. Un ejemplo de conflicto puede estar representado por lo que sucedió el 11 de abril de 2019, cuando los gobiernos de Argentina, Brasil, Chile, Colombia y Paraguay presentaron al Secretario Ejecutivo de la Comisión Interamericana de Derechos Humanos (CIDH) una Declaración conjunta sobre el estado actual del sistema interamericano en la que, según afirmaban, «se debe respetar el legítimo espacio de autonomía del que disponen los Estados» ${ }^{43}$. La puesta en entredicho de la autoridad de las organizaciones internacionales, y no solo de los tribunales internacionales, ha sido un fil rouge también de la actuación de la actual administración estadounidense, que desde hace meses está dificultando o paralizando la acción de la Organización Mundial del Comercio, y en julio llegó a notificar a las Naciones Unidas su salida de la Organización Mundial de la Salud por su rol en la pandemia.

42 Disponible en: https://www.imf.org/es/Topics/imf-and-covid19.

43 Disponible en: https://bit.ly/34yUkWt. 
Pero tampoco en la UE corren buenos tiempos para la integración supranacional y, en este contexto, la sentencia del TCFA que se ha venido analizando constituye también un peligroso ejemplo para otros tribunales, cuyas decisiones futuras a imitación del Alto Tribunal alemán pueden suponer un riesgo para la propia subsistencia de la UE en un momento histórico en el que se enfrenta a fuertes movimientos centrípetos provenientes de distintos Estados miembros como Polonia y Hungría (respecto de los cuales, junto a la República Checa), el TJUE acaba de declarar el incumplimiento de las obligaciones derivadas de las Decisiones relativas a la reubicación de solicitantes de protección internacional $\left.{ }^{44}\right)$. ¿Qué pasaría si considerasen que la sentencia no está bien argumentada y la declararan inaplicable, siguiendo el ejemplo de la decisión alemana?

En todo caso, más allá de las repercusiones de la sentencia del TCFA en el ordenamiento jurídico y en la organización institucional de la UE, como subraya M. Aragón Reyes (2019: 37-38), en la actual situación de globalización, universalización y supraestatalización en materia de derechos humanos, el futuro de la justicia constitucional «se juega» en el terreno de su imbricación en el «sistema de justicia constitucional supraestatal» a través del mantenimiento de un continuo diálogo entre jurisdicciones.

\section{Bibliografía}

Amtenbrink, F. (2019). The European Central Bank's intricate independence versus accountability conundrum in the post-crisis governance framework. Maastricht Journal of European and Comparative Law, 26(1), 165-179. Disponible en: https://doi.org/10.1177/1023263X18822789.

Aragón Reyes, M. (2019). El futuro de la justicia constitucional. Anuario Iberoamericano de Justicia Constitucional, 23(1), 11-41. Disponible en: https://doi. org/10.18042/cepc/aijc.23.01.

Beukers, T. (2013). The New ECB and its Relationship with the Eurozone Member States: Between Central Bank Independence and Central Bank Intervention. Common Market Law Review, 50, 1579-1620.

Bini Smaghi, L. y Casini, C. (2000). Monetary and Fiscal Policy Co-operation: Institutions and Procedures in EMU. Journal of Common Market Studies, 38(3), 375-391. Disponible en: https://doi.org/10.1111/1468-5965.00226.

Cafaro, S. (2020). Quale Quantitative Easing e quale Unione europea dopo la sentenza del 5 maggio? Sidiblog.org, 8 de mayo. Disponible en: https://bit.ly/3mnWXAq.

44 Sentencia de 2 de abril de 2020, Comisión c. Polonia, Hungría y República Checa, asuntos acumulados C-715/17, C-718/17 y C-719/17. 
Caponi, R. (2014). La Corte costituzionale tedesca e la crisi dell'eurozona. Salvaguardare l'euro con ogni mezzo? Il primo rinvio pregiudiziale della Corte costituzionale tedesca. Giornale di Diritto amministrativo, 5, 469-478.

Caravita, B., Condinanzi, M., Morrone, A., y Poggi, A. M. (2020). Da Karlsruhe una decisione poco meditata in una fase politica che avrebbe meritato maggiore ponderazione. Federalismi.it, 4-14.

Ciancio, A. (2020). The 5 May 2020 Bundesverfassungsgericht's Decision on the ECB's public sector purchase program: an attempt to «break the toy'» or a new starting point for the Eurozone? Federalismi.it, 35-47.

Committee for the Study of Economic and Monetary Union. Jacques Delors Chairman (1989). Report on Economic and Monetary Union in the European Community, 1-40.

Conclusiones Consejo Europeo (2010). EUCO 7/10, 25-26 marzo, 1-11.

De Grauwe, P. (2012). The European Central Bank: Lender of Last Resort in the Government Bond Markets. En F. Allen et al. (eds.). Governance for the Eurozone. Integration or Disintegration? Philadelphia: FIC Press.

De Haan, J., Amtenbrink, F. y Waller, S. (2004). The transparency and Credibility of the European Central Bank. Journal of Common Market Studies, 42(4), 775794. Disponible en: https://doi.org/10.1111/j.0021-9886.2004.00529.x.

De Vergottini, G. (2010). Más allá del diálogo entre tribunales. Comparación y relación entre jurisdicciones. Madrid: Cuadernos Civitas. Disponible en: https://doi. org/10.5944/trc.28.2011.6962.

Duisenberg, W. F. (2005). Introduction. En Banco Central Europeo. The Eurosystem, the Union and Beyond. The Single Currency and Implications for Governance. Disponible en: https://bit.ly/31I31KF.

ECB (2010). The ECB's relations with European Union institutions and bodies - trends and prospects. Monthly Bulletin January, 73-84.

Fabbrini, F. (2016). Guest Editor's Introduction. The European Court of Justice, the European Central Bank, and the Supremacy of EU Law. Maastricht Journal of European and Comparative Law, 23(1), 3-16. Disponible en: https://doi.org/10.1177/1023263X1602300101.

Faraguna, P. (2020). Il Bundesverfassungsgericht dichiara ultra vires il Quantitative easing (con uno sguardo al Pandemic Emergency Purchase Programme?). Forum di Quaderni Costituzionali, 2, 306-315.

Follesdal, A. y Hix, S. (2006). Why There is a Democratic Deficit in the EU: A Response to Majone and Moravcsik. Journal of Common Market Studies, 44(3), 533-562. Disponible en: https://doi.org/10.1111/j.1468-5965.2006.00650.x.

Fromage, D., Dermine, P., Nicolaides, P. y Tuori, K. (2019). ECB independence and accountability today: Towards a (necessary) redefinition? Maastricht Journal of European and Comparative Law, 26(1), 3-16. Disponible en: https://doi. org/10.1177/1023263X19827819.

Galetta, D. U. y Ziller, J. (2020). «¿Karlsruhe über alles?» la insoportable pesadez del juez constitucional alemán (sobre la sentencia del «Zweiter Senat» del Tribunal Constitucional Federal alemán del 5 de mayo de 2020 relativa al programa PSPP del Banco Central Europeo). Revista Española de Derecho Europeo, 73-74, 179212. Disponible en: https://doi.org/10.37417/REDE/num73-74_2020_390. 
González Pascual, M. (2010). El Tribunal Constitucional alemán en la construcción del espacio europeo de los derechos. Cizur Menor: Aranzadi.

Grimm, D. (2020). A Long Time Coming. German Law Journal, 21(5), 944-949. Disponible en: https://doi.org/10.1017/glj.2020.55.

Hahn, H. J. (1991). The European Central Bank: Key to European Monetary Union or Target? Common Market Law Review, 28(4), 783-820.

Hinarejos, A. (2015). Gauweiler and the Outright Monetary Transactions Programme: The Mandate of the European Central Bank and the Changing Nature of Economic and Monetary Union. European Court of Justice, Judgement of 16 June 2015, Case C-62/14 Gauweiler and others v. Deutscher Bundestag. European Constitutional Law Review, 11, 563-576. Disponible en: https://doi. org/10.1017/S1574019615000346.

Högenauer,A-L.y Howarth,D.(2019).ThedemocraticdeficitandEuropean Central Bank crisis monetary policies. Maastricht Journal of European and Comparative Law, 26(1), 81-93. Disponible en: https://doi.org/10.1177/1023263X18824776.

Jędrzejowska-Schiffauer, I. y Schiffauer, P. (2016). European Central Bank's OMT decision: still within the framework of the monetary policy? Journal of International Studies, 9(2), 195-206. Disponible en: https://doi.org/10.14254/2071$8330.2016 / 9-2 / 15$.

Jiménez-Blanco y Carrillo de Albornoz, A. (2020). La compra de deuda pública por el Banco Central Europeo: notas sobre la Sentencia del Tribunal Constitucional Federal de Alemania de 5 de mayo de 2020. Revista de Administración Pública, 212, 147-179. Disponible en: https://doi.org/10.18042/cepc/rap.212.05.

Lozano Cutanda, B. (2020). La sentencia del Tribunal Constitucional Federal alemán: ¿y ahora qué? Análisis $G A \_P$, mayo, 1-6.

Majone, G. (1998). Europe's 'Democratic deficit': The Question of Standards. European Law Journal, 4(1), 5-28. Disponible en: https://doi.org/10.1111/14680386.00040 .

Mangas Martín, A. (2020). El Tribunal Constitucional Alemán y su «fuego amigo» sobre el Tribunal de Justicia de la UE y el BCE. ARI Real Instituto Elcano 72/2020, 1-15. Disponible en: https://bit.ly/31KVegq.

Mooij, A. (2019). The Weiss judgement: The Court's further clarification of the ECB's legal framework. Maastricht Journal of European and Comparative Law, 26(3), 449-465. Disponible en: https://doi.org/10.1177/1023263X19837208.

Moravcsik, A. (2002). In defense of the 'Democratic Deficit': Reassessing Legitimacy in the European Union. Journal of Common Market Studies, 40(4), 5-28. Disponible en: https://doi.org/10.1111/1468-5965.00390.

Pace, L. F. (2020). Il BVerGe la sentenza sul programma PSPP: «c'è della logica in questa follia»? Il prevedibile «rientro» della "crisi istituzionale» annunciata nella sentenza (provvisoria) del 5 maggio 2020. Federalismi.it, 299-309.

Poiares Maduro, M. (2020). Some Preliminary Remarks on the PSPP Decision of the German Constitutional Court. Verfassungsblog: On Matters Constitutional [blog], 6-5-2020. Disponible en: https://bit.ly/3jBz1b2.

Ragone, S. (2011). Las relaciones de los Tribunales Constitucionales de los Estados miembros con el Tribunal de Justicia y con el Tribunal Europeo de Derechos 
Humanos: una propuesta de clasificación. Revista de Derecho Constitucional Europeo, 16, 53-90.

Roca, M. J. (2020). La sentencia del Tribunal Constitucional Federal Alemán sobre el Programa de Compra de Bonos por el Banco Central Europeo: el control ultra vires y la primacía del Derecho Europeo. DPCE online, 2, 2845-2856.

Rovetta, D. y Smiatacz, A. (2020). The German Federal Constitutional Court's Judgment on the PSPP: What happened to the EU law Supremacy? Implications of the Judgment for the Trade and Customs Policy of the European Union. Global Trade and Customs Journal, 15(9), 435-444.

Sáinz de Vicuña y Barroso, A. (2016). La política monetaria del BCE ante el Tribunal Constitucional Federal alemán: la sentencia de 21 de junio de 2016 en el caso "OMT». Revista de Derecho Comunitario Europeo, 55, 1067-1099. Disponible en: https://doi.org/10.18042/cepc/rdce.55.08.

Schneider, K. (2020). Gauging «Ultra-Vires»: The Good Parts. German Law Journal, 21(5), 968-978. Disponible en: https://doi.org/10.1017/glj.2020.61.

Simon, S. (2015). Direct Cooperation Has Begun: Some Remarks on the Judgement of the ECJ on the OMT Decision of the ECB in Response to the German Federal Constitutional Court's First Request for a Preliminary Ruling. German Law Journal, 16(4), 1025-1048. Disponible en: https://doi.org/10.1017/ S2071832200019982.

Smits, R. (1991). The European Central Bank: Institutional Aspects. International and Comparative Law Quarterly, 45, 319-342. Disponible en: https://doi. org/10.1017/S0020589300059017.

- (2008). The European Central Bank's Independence and Its Relations with Economic Policy Makers. Fordham International Law Journal, 31, 1614-1636.

- (2012). Correspondence. Common Market Law Review, 49(2), 827-831.

Tesauro, G. y De Pasquale, P. (2020). La BCE e la Corte di giustizia sul banco degli accusati del Tribunale costituzionale tedesco. Il Diritto dell'Unione Europea - Osservatorio Europeo, 11 de mayo. Disponible en: https://bit.ly/3omH1Ad.

Wendel, M. (2020). Paradoxes of Ultra-Vires Review: A Critical Review of the PSPP Decision and Its Initial Reception. German Law Journal, 21(5), 979-994. Disponible en: https://doi.org/10.1017/glj.2020.62.

Wolff, G. B. (2013). The ECB's OMT Programme and German Constitutional Concerns. En I. Atiyas et al., The G-20 and Central Banks in the New World of Unconventional Monetary Policy (pp. 26-31). Disponible en: https://brook. gs/35FZLCi.

Ziller, J. (2010). Zur Europarechtsfreundlichkeit des deutschen Bundesverfassungsgerichtes. Eine ausländische Bewertung des Urteils des Bundesverfassungsgerichtes zur Ratifikation des Vertrages von Lissabon. Zeitschrift für öffentliches Recht, 65, 157-176. Disponible en: https://doi.org/10.1007/s00708-010-0046-2. 